\title{
Defining velocities for accurate kinetic statistics in the GJF thermostat
}

\author{
Niels Grønbech-Jensen ${ }^{1,2}$ and Oded Farago ${ }^{3,4}$ \\ ${ }^{1}$ Department of Mechanical \&S Aerospace Engineering, \\ University of California, Davis, CA 95616, USA \\ ${ }^{2}$ Department of Mathematics, University of California, Davis, CA 95616, USA \\ ${ }^{3}$ Department of Chemistry, University of Cambridge, Cambridge CB2 1EW, United Kingdom \\ ${ }^{4}$ Department of Biomedical Engineering, Ben Gurion University of the Negev, Be'er Sheva, 84105 Israel
}

\begin{abstract}
We expand on two previous developments in the modeling of discrete-time Langevin systems. One is the well-documented Grønbech-Jensen Farago (GJF) thermostat, which has been demonstrated to give robust and accurate configurational sampling of the phase space. Another is the recent discovery that also kinetics can be accurately sampled for the GJF method. Through a complete investigation of all possible finite difference approximations to the velocity, we arrive at two main conclusions: 1) It is not possible to define a so-called on-site velocity such that kinetic temperature will be correct and independent of the time step, and 2) there exists a set of infinitely many possibilities for defining a two-point (leap-frog) velocity that measures kinetic energy correctly for linear systems in addition to the correct configurational statistics obtained from the GJF algorithm. We give explicit expressions for the possible definitions, and we incorporate these into convenient and practical algorithmic forms of the normal Verlet-type algorithms along with a set of suggested criteria for selecting a useful definition of velocity.
\end{abstract}

\section{INTRODUCTION}

Simulations of statistical properties in complex systems have been a subject of intense interest for the past several decades [1 3], especially in the area of Molecular Dynamics, where thermodynamic ensembles are sampled by following the temporal evolution of large numbers of interacting particles. This is done by numerically integrating Newton's equation of motion of each particle. The most commonly used algorithm for this purpose is the one proposed by Verlet [4], which is correct to second order in the integration time step, $d t$, and conserves energy in long-time integrations. The Verlet algorithm samples the microcanonical ensemble, but the more frequently used ensemble in statistical-mechanics is the canonical $(N, V, T)$ ensemble where the temperature of the system, rather than its energy, is constant. Many methods for controlling the temperature of a simulated system (thermostats) have been developed, and most of them fall into two major categories: Deterministic (e.g., Nosé-Hoover [5, 6]) and stochastic (Langevin) thermostats [7-16]. The deterministic approach includes additional degrees of freedom, which act as an energy reservoir and thereby mimic a thermal heat bath. A requirement for such method is that the temperature of a simulated system can be reliably measured in order for the system to interact properly with the heat-bath. The stochastic approach is based on the assumption that each particle in the system has its behavior modeled by a Langevin equation [17]

$$
m \dot{v}+\alpha \dot{r}=f+\beta,
$$

where $m$ is the mass of an object with spatial coordinate, $r$, and velocity $v=\dot{r}$, and $f$ is the force acting on the coordinate. This is Newton's second law with two additional terms representing the interactions with a heat bath: (i) Linear (in $v$ ) friction, which is represented by the friction constant $\alpha \geq 0$, and stochastic white noise, $\beta(t)$, which can be chosen to be a Gaussian distributed variable. These terms are thermodynamically matched through the fluctuation-dissipation theorem by [18]

$$
\begin{aligned}
\langle\beta(t)\rangle & =0 \\
\left\langle\beta(t) \beta\left(t^{\prime}\right)\right\rangle & =2 \alpha k_{B} T \delta\left(t-t^{\prime}\right),
\end{aligned}
$$

where $k_{B}$ is Boltzmann's constant and $T$ is the thermodynamic temperature.

Integrating numerically a Langevin equation of motion poses a challenge since discrete time tends to distort the conjugated relationship between the positional coordinate and its corresponding momentum (see Appendix in Ref. [19]). A resulting problem is that the kinetic and configurational measures of temperature disagree, which is a concern for both the integrity of a simulation and the extraction of self-consistent information that may depend on configurational as well as kinetic sampling. It is therefore imperative to understand how to properly define a kinetic measure consistent with the statistics from the trajectory. We note that the issue of defining configurational temperatures for also investigating nonequilibrium ensembles have been extensively pursued in, e.g., Refs. 20 23]. As our concern in this paper is to devise kinetically predictable velocity definitions that accompany the GJF method in equilibrium, we will not directly be addressing the configurational temperature, since the GJF method is already known to provide timestep independent configurational sampling in equilibrium [15, 24].

The possibility of creating a discrete-time simulation method that gives statistically sound results for both configurational and kinetic measures was first reported in Ref. [19], where the statistically correct 2GJ half-step velocity was identified, and comprehensively demonstrated to give robust (i.e., independent of the integration time step, $d t$, for the entire stability range of time-steps) 
statistics for both nonlinear and complex molecular systems. The algorithm, which is rooted in the statistically sound spatial trajectory of the GJF algorithm [15, 24, 25], is formulated in a typical Leap-Frog (LF) form that is easily implemented into existing Molecular Dynamics codes. Subsequently, a related formulation of the GJF algorithm, with similar kinetic averages, has been identified [26]. It is the objective of this paper to demonstrate that there exists a large set of kinetically correct velocity definitions given by one free parameter, and that this set includes the reported velocities [19, 26].

\section{DISCRETE-TIME LANGEVIN DYNAMICS}

Since the starting point of this work is the spatial GJF trajectory, we give a brief review of the features of this method here. The GJF algorithm for simulating Eq. (1) in discrete time is [15]

$$
\begin{aligned}
& r^{n+1}=r^{n}+b\left[d t v^{n}+\frac{d t^{2}}{2 m} f^{n}+\frac{d t}{2 m} \beta^{n+1}\right] \\
& v^{n+1}=a v^{n}+\frac{d t}{2 m}\left(a f^{n}+f^{n+1}\right)+\frac{b}{m} \beta^{n+1},
\end{aligned}
$$

where $r^{n}, v^{n}$, and $f^{n}$ are the discrete-time GJF position, velocity, and force, respectively, at time $t_{n}$, and where

$$
\begin{aligned}
& a=\frac{1-\frac{\alpha d t}{2 m}}{1+\frac{\alpha d t}{2 m}} \\
& b=\frac{1}{1+\frac{\alpha d t}{2 m}}
\end{aligned}
$$

are the coefficients that define the discrete-time attenuation. The associated discrete-time noise is

$$
\beta^{n+1}=\int_{t_{n}}^{t_{n+1}} \beta\left(t^{\prime}\right) d t^{\prime},
$$

which results in an uncorrelated Gaussian random variable with zero mean and a variance given by the temperature and friction coefficient:

$$
\begin{aligned}
\left\langle\beta^{n}\right\rangle & =0 \\
\left\langle\beta^{n} \beta^{l}\right\rangle & =2 \alpha k_{B} T d t \delta_{n, l},
\end{aligned}
$$

where $\delta_{n, l}$ is the Kronecker delta function.

As was pointed out in Ref. [15], the basic thermodynamic properties for a flat potential, $f=0$ are well behaved. The equipartition theorem for the kinetic energy is satisfied:

$$
\left\langle E_{k}\right\rangle=\frac{1}{2} m\left\langle\left(v^{n}\right)^{2}\right\rangle=\frac{1}{2} k_{B} T,
$$

and the configurational Einstein diffusion

$$
D_{E}=\lim _{n d t \rightarrow \infty} \frac{\left\langle\left(r^{n}-r^{0}\right)^{2}\right\rangle}{2 n d t}=\frac{k_{B} T}{\alpha},
$$

yields the correct expectation for any set of simulation parameters, including the time step. Appendix A shows that also the Green-Kubo evaluation of diffusion can yield the correct value $D_{E}$ if a particular Riemann approximation is applied to the Green-Kubo integral.

Notice that the velocity attenuation factor $a(|a|<1)$ in Eq. (6) is negative for time steps larger than

$$
d t_{a}=2 m / \alpha .
$$

Choosing $d t>d t_{a}$ does not affect the robust configurational sampling properties of the GJF method [15], but (as will be discussed below) it may lead to certain nonphysical features of the discrete-time velocity autocorrelation.

\section{A. GJF for Linear Systems, $f=-\kappa r$}

While the kinetic measure of diffusion in a flat potential can be defined correctly for the GJF velocity variable, the harmonic oscillator, given by $f^{n}=-\kappa r^{n}$ with $\kappa>0$, shows how configurational and kinetic statistics are no longer mutually consistent when the potential has curvature. In [15], we showed that for $n \rightarrow \infty$

$$
\begin{aligned}
& \left\langle E_{p}\right\rangle=\frac{1}{2} \kappa\left\langle\left(r^{n}\right)^{2}\right\rangle=\frac{1}{2} k_{B} T \\
& \left\langle E_{k}\right\rangle=\frac{1}{2} m\left\langle\left(v^{n}\right)^{2}\right\rangle=\frac{1}{2} k_{B} T\left(1-\frac{\left(\Omega_{0} d t\right)^{2}}{4}\right),
\end{aligned}
$$

where $\Omega_{0}=\sqrt{\kappa / m}$ is the natural frequency of the oscillator. These results hold for any time step smaller than the Verlet stability limit $\Omega_{0} d t \leq 2$. The appealing features of the GJF algorithm is given by Eqs. (12) and (14) as these indicate sound results for configurational statistics, which is the aim of computer simulation studies of, e.g., molecular systems at equilibrium. As promoted in Ref. 19], the discrete-time velocity is predominantly an auxiliary variable, which for simulations of equilibrium is used primarily for assessing the temperature of the simulated system via the mean kinetic energy. Since $\Omega_{0}$ is an expression of the curvature of the potential, Eq. (15) shows that a general system cannot be simulated with a correct kinetic statistical measure using the GJF velocity Eq. (5).

With the useful GJF spatial trajectory and the accompanying depressed on-site GJF velocity, $v^{n}$, which results in imperfect kinetic statistics, we here investigate the kinetic response of all finite difference velocities.

\section{B. A general finite difference velocity}

Since the aim of this section is to explore velocity definitions that may accompany the GJF trajectory, it is natural to write the GJF method in its Størmer-Verlet 
form [19, 24]:

$$
r^{n+1}=2 b r^{n}-a r^{n-1}+\frac{b d t^{2}}{m} f^{n}+\frac{b d t}{2 m}\left(\beta^{n}+\beta^{n+1}\right),
$$

with the GJF velocity Eq. (5) expressed as [19]

$$
v^{n}=\frac{r^{n+1}-(1-a) r^{n}-a r^{n-1}}{2 b d t}+\frac{\beta^{n}-\beta^{n+1}}{4 m} .
$$

Inspired by Eq. (17), we define a velocity $w$ in the general finite-difference form

$$
w=\frac{\gamma_{1} r^{n+1}+\gamma_{2} r^{n}+\gamma_{3} r^{n-1}}{d t}+\frac{\gamma_{4} \beta^{n}+\gamma_{5} \beta^{n+1}}{m},
$$

where $\gamma_{i}$ are unit-less constants that are to be determined, and where the two noise terms, $\beta^{n}$ and $\beta^{n+1}$, span the time interval of the finite difference, $t_{n-1}<t<t_{n+1}$ [see Eq. [8] ]. Notice that we have not attached a superscript on $w$ that indicates at which time this velocity is represented, since this general expression is representing any velocity approximation in the interval spanned by the finite difference. Specifically, we recognize the usual three-point on-site and two-point half-step velocities in the frictionless $(\alpha=0)$ Verlet algorithm

$$
\begin{aligned}
v^{n} & =\frac{r^{n+1}-r^{n-1}}{2 d t} \\
v^{n+\frac{1}{2}} & =\frac{r^{n+1}-r^{n}}{d t},
\end{aligned}
$$

for $\gamma_{1}=-\gamma_{3}=\frac{1}{2}, \gamma_{2}=\gamma_{4}=\gamma_{5}=0$ and $\gamma_{1}=-\gamma_{2}=1$, $\gamma_{3}=\gamma_{4}=\gamma_{5}=0$, respectively. We also recognize the GJF velocity, $v^{n}$, in Eq. (17), as represented by $\gamma_{1}=$ $1 / 2 b, \gamma_{2}=-(1-a) / 2 b, \gamma_{3}=-a / 2 b, \gamma_{4}=-\gamma_{5}=1 / 4$.

We start by writing the most basic statistical requirement to a velocity variable, namely

$$
\langle w w\rangle=\frac{k_{B} T}{m} .
$$

Using Eqs. (16) and (18), $\langle w w\rangle$ can be rewritten

$$
\begin{aligned}
\langle w w\rangle & =\frac{\gamma_{1}^{2}+\gamma_{2}^{2}+\gamma_{3}^{2}}{d t^{2}}\left\langle r^{n} r^{n}\right\rangle+2 \frac{\gamma_{1} \gamma_{2}+\gamma_{2} \gamma_{3}}{d t^{2}}\left\langle r^{n} r^{n+1}\right\rangle \\
& +2 \frac{\gamma_{1} \gamma_{3}}{d t^{2}}\left\langle r^{n-1} r^{n+1}\right\rangle+\frac{\gamma_{4}^{2}+\gamma_{5}^{2}}{m^{2}}\left\langle\beta^{n} \beta^{n}\right\rangle \\
& +2 \frac{\gamma_{1} \gamma_{4}}{m d t}\left\langle r^{n+1} \beta^{n}\right\rangle+2 \frac{\gamma_{1} \gamma_{5}+\gamma_{2} \gamma_{4}}{m d t}\left\langle r^{n} \beta^{n}\right\rangle .
\end{aligned}
$$

From Eqs. (10), (14), and (16) we obtain the relevant correlations:

$$
\begin{aligned}
\left\langle\beta^{n} r^{n}\right\rangle & =(b-a) k_{B} T d t \\
\left\langle\beta^{n} r^{n+1}\right\rangle & =\left(2+a-b \Omega_{0}^{2} d t^{2}\right)(b-a) k_{B} T d t \\
\left\langle r^{n} r^{n+1}\right\rangle & =\frac{k_{B} T}{\kappa}\left(1-b \frac{\Omega_{0}^{2} d t^{2}}{2}\right) \\
\left\langle r^{n-1} r^{n+1}\right\rangle & =\frac{k_{B} T}{\kappa}\left(1-b(b+1) \Omega_{0}^{2} d t^{2}+b^{2} \frac{\Omega_{0}^{4} d t^{4}}{2}\right),
\end{aligned}
$$

which, when inserted into Eq. (22), yield

$$
\begin{aligned}
& \frac{m\langle w w\rangle}{k_{B} T}=\frac{\gamma_{1}^{2}+\gamma_{2}^{2}+\gamma_{3}^{2}+2\left(\gamma_{1} \gamma_{2}+\gamma_{2} \gamma_{3}+\gamma_{3} \gamma_{1}\right)}{\Omega_{0}^{2} d t^{2}} \\
& -b\left[\gamma_{1} \gamma_{2}+\gamma_{2} \gamma_{3}+2(1+b) \gamma_{3} \gamma_{1}\right]+4\left(\gamma_{4}^{2}+\gamma_{5}^{2}\right) \frac{1-b}{b} \\
& +4(1-b)\left[\gamma_{1} \gamma_{5}+\gamma_{2} \gamma_{4}+(1+2 b) \gamma_{1} \gamma_{4}\right] \\
& +\Omega_{0}^{2} d t^{2} b \gamma_{1}\left[b \gamma_{3}-4(1-b) \gamma_{4}\right] .
\end{aligned}
$$

While this expression is somewhat cumbersome, it immediately reveals key information about possible definitions of kinetically robust velocities to accompany the G.JF trajectory. First, from the requirement that Eq. (21) is satisfied for any (stable) $d t$, it follows that the terms in Eq. (27) proportional to both $\left(\Omega_{0} d t\right)^{-2}$ and $\left(\Omega_{0} d t\right)^{2}$ must be zero. Thus, we must require that

$$
\begin{aligned}
& 0=\gamma_{1}^{2}+\gamma_{2}^{2}+\gamma_{3}^{2}+2\left(\gamma_{1} \gamma_{2}+\gamma_{2} \gamma_{3}+\gamma_{3} \gamma_{1}\right), \\
& 0=b \gamma_{1}\left[b \gamma_{3}-4(1-b) \gamma_{4}\right] .
\end{aligned}
$$

Second, since $w$ represents the velocity during the time interval $t_{n} \leq t<t_{n+1}$, we will further require that $\gamma_{1} \neq$ 0 . Moreover, in the limit $\alpha d t \rightarrow 0\left(a, b \rightarrow 1, \beta^{n}=\right.$ $\left.\beta^{n+1}=0\right)$ the coefficient $\gamma_{1}$ should become either $\gamma_{1} \rightarrow \frac{1}{2}$ or $\gamma_{1} \rightarrow 1$, such that $w$ becomes one of the two known velocities given in Eqs. (19) and (20) in that limit. Under these conditions, Eq. (29) yields the following noise term associated with $\beta^{n}$ :

$$
\gamma_{4} \beta^{n}=\frac{b}{1-b} \gamma_{3} \sqrt{2 \alpha k_{B} T d t} \sigma^{n}=2 m \gamma_{3} \sqrt{2 \frac{k_{B} T}{\alpha d t}} \sigma^{n},
$$

where $\sigma^{n} \in N(0,1)$ is a random number with a standard normal distribution. The requirement for $\gamma_{4}$ to be confined is that $\gamma_{3} \rightarrow 0$ faster than $(\alpha d t)^{\frac{1}{2}}$ for $\alpha d t \rightarrow 0$. This condition, however, cannot be met by on-site velocity variables $v^{n}$, which in the limit $\alpha d t \rightarrow 0$ must coincide with Eq. (19), where $\gamma_{1}=-\gamma_{3}=\frac{1}{2} \neq 0$, and the limit $\gamma_{3} \rightarrow-\frac{1}{2}$ would create a diverging noise term in any on-site velocity definition as $\alpha d t \rightarrow 0$. We therefore conclude that no reasonable on-site finite-difference velocity that has correct and time step independent kinetic statistics can be defined such that it will approach the expected central difference approximation in the limit $\alpha d t \rightarrow 0$.

\section{Two-point velocity, $\gamma_{3}=\gamma_{4}=0$}

In light of Eq. (20), we will throughout this paper denote a two-point velocity $w=u^{n+\frac{1}{2}}$ as one given by Eq. (18) with $\gamma_{3}=\gamma_{4}=0$, such that the value of the denoted two-point velocity pertains to the time interval $t_{n}<t<t_{n+1}$.

Setting $\gamma_{3}=\gamma_{4}=0$, considerately simplifies Eq. (27) to

$$
\begin{aligned}
& \frac{m\left\langle\left(u^{n+\frac{1}{2}}\right)^{2}\right\rangle}{k_{B} T}=\frac{\left(\gamma_{1}+\gamma_{2}\right)^{2}}{\Omega_{0}^{2} d t^{2}} \\
& -b \gamma_{1} \gamma_{2}+4 \gamma_{5}^{2} \frac{1-b}{b}+4(1-b) \gamma_{1} \gamma_{5} .
\end{aligned}
$$


With the requirement that Eq. (21) holds for any $d t$, we have $\gamma_{1}=-\gamma_{2}$, which then yields the condition:

$$
b \gamma_{1}^{2}+4 \frac{1-b}{b} \gamma_{5}^{2}+4(1-b) \gamma_{1} \gamma_{5}=1 .
$$

From this expression, we can determine $\gamma_{5}$ as a function of a given $\gamma_{1}$ :

$$
\gamma_{5}=-\frac{1}{2} b \gamma_{1} \pm \frac{1}{2} \sqrt{b \frac{1-b^{2} \gamma_{1}^{2}}{1-b}},
$$

which implies that $\left(b \gamma_{1}\right)^{2} \leq 1$. We will also require that $\gamma_{1} \rightarrow 1$ for $b \rightarrow 1$ in order to recover the corresponding velocity in the limit of either continuous time or no friction, $\alpha d t \rightarrow 0$ [see discussion above, after Eq. (29)]. Thus, we have identified a family of velocities that yield the correct average kinetic energy in discrete-time:

$$
u^{n+\frac{1}{2}}=\gamma_{1} \frac{r^{n+1}-r^{n}}{d t}+\frac{\gamma_{5}}{m} \beta^{n+1},
$$

where $\gamma_{5}$ is determined by the parameter $\gamma_{1}$, which is limited in magnitude by $\left|b \gamma_{1}\right| \leq 1$.

Using Eq. (34) for the velocity, together with Eq. (16) for the GJF trajectory, we arrive for $\gamma_{1} \neq 0$ at the following general LF GJF algorithm:

$$
\begin{aligned}
u^{n+\frac{1}{2}} & =a u^{n-\frac{1}{2}}+\gamma_{1} \frac{b d t}{m} f^{n}+\frac{\Gamma_{4}}{2 m} \beta^{n}+\frac{\Gamma_{5}}{2 m} \beta^{n+1} \\
r^{n+1} & =r^{n}+\frac{d t}{\gamma_{1}} u^{n+\frac{1}{2}}-\frac{\gamma_{5}}{\gamma_{1}} \frac{d t}{m} \beta^{n+1}
\end{aligned}
$$

where

$$
\begin{aligned}
& \Gamma_{4}=b \gamma_{1}-2 a \gamma_{5} \\
& \Gamma_{5}=b \gamma_{1}+2 \gamma_{5} .
\end{aligned}
$$

The general scheme Eqs. (35)-(38) can be also written in the following form, involving both the denoted two-point velocity, $u^{n+1 / 2}$, and the on-site velocity, $v^{n}$, expressed in Eqs. (5) and (17):

$$
\begin{aligned}
u^{n+\frac{1}{2}} & =b \gamma_{1}\left[v^{n}+\frac{1}{2 m} \frac{\Gamma_{5}}{b \gamma_{1}} \beta^{n+1}+\frac{d t}{2 m} f^{n}\right] \\
r^{n+1} & =r^{n}+\frac{d t}{\gamma_{1}} u^{n+\frac{1}{2}}-\frac{\gamma_{5}}{\gamma_{1}} \frac{d t}{m} \beta^{n+1} \\
v^{n+1} & =\frac{a}{b \gamma_{1}} u^{n+\frac{1}{2}}+\frac{1}{2 m} \frac{\Gamma_{4}}{b \gamma_{1}} \beta^{n+1}+\frac{d t}{2 m} f^{n+1} .
\end{aligned}
$$

This compact form of the method further illuminates the meaning of the parameter $\gamma_{1}$ beyond the direct scaling of the finite-difference two-point velocity, observed in Eq. (34). As mentioned at the beginning of section [I] the total attenuation factor of the velocity over one time step is $a$, and this factor is shown in Eqs. (39)-(41) to be partitioned into two parts: The first is the attenuation $b \gamma_{1}$ of the velocity $v^{n}$ into the velocity $u^{n+\frac{1}{2}}$; the second is the attenuation $a / b \gamma_{1}$ of the velocity $u^{n+\frac{1}{2}}$ into $v^{n+1}$. The product of the two attenuation factors is obviously $a$. It is physically reasonable to expect that the attenuation factor is positive and not larger than unity in either of the two parts of the time step. A negative attenuation factor $b \gamma_{1}$ implies a peculiar velocity that is in directional opposition to the surrounding trajectory, whereas a factor which is greater than unity implies velocity amplification. Thus, in order to have a physically meaningful description of the velocity attenuation, we must choose (i) $a \geq 0\left[d t \leq d t_{a}\right.$ - see discussion around Eq. [13)], and (ii) $a \leq b \gamma_{1} \leq 1$. With that said, we reemphasize that any velocity defined by Eq. (34) will always yield the correct average of the kinetic energy, and that this form only requires that $\left|b \gamma_{1}\right| \leq 1$.

\section{Special cases}

We now highlight the following three choices of $\gamma_{1}$ as examples of velocity definitions:

Case A: $\sqrt{b} \gamma_{1}=1, \gamma_{5}=0$. This is the 2GJ half-step velocity given in Ref. [19],

$$
u_{A}^{n+\frac{1}{2}}=\frac{r^{n+1}-r^{n}}{\sqrt{b} d t},
$$

and it is the optimal amplitude rescaling $\gamma_{1}$ of the standard definition Eq. (201), since it is the only form where $\gamma_{5}=0$; i.e., the only form where the symmetry of the central difference is not broken by an additional noise contribution in order to yield the correct kinetic energy. The coefficients to the noise terms in Eq. (35) are given by $\Gamma_{4}=\Gamma_{5}=\sqrt{b}$.

Case B: $b \gamma_{1}=1, \gamma_{5}=-\frac{1}{2}$. This velocity is given in Ref. [26]:

$$
u_{B}^{n+\frac{1}{2}}=\frac{r^{n+1}-r^{n}}{b d t}-\frac{1}{2 m} \beta^{n+1},
$$

and is the maximum amplitude rescaling $\gamma_{1}$ of the standard definition Eq. (20). It includes an explicit noise contribution in order to achieve the correct kinetic energy statistics. The velocity attenuation is here assigned exclusively to the second half of the time step as seen from Eqs. (39)-(41). The coefficients to the noise terms in Eq. (35) are given by $\Gamma_{4}=2 b$ and $\Gamma_{5}=0$.

Case C: $\gamma_{1}=1, \gamma_{5}=-\frac{1}{2}(b-\sqrt{b(1+b)})$. This case defines the velocity from the neutral amplitude rescaling $\gamma_{1}=1$ of the standard definition Eq. (20), which is

$$
u_{C}^{n+\frac{1}{2}}=\frac{r^{n+1}-r^{n}}{d t}-\frac{1}{2 m}(b-\sqrt{b(1+b)}) \beta^{n+1} .
$$

While this expression has a non-trivial pre-factor $\gamma_{5}$ to the compensating noise term, it may be attractive by the absence of amplitude scaling of the velocity 
Eq. (20). Thus, the average velocity is here correctly representing a ballistic (constant velocity) trajectory. The coefficients to the noise terms in Eq. (35) are given by $\Gamma_{4}=2 b^{2}-a \sqrt{b(1+b)}$ and $\Gamma_{5}=\sqrt{b(1+b)}$.

We re-emphasize that simply obtaining the correct kinetic energy is not a sufficient criterion for a physically reasonable definition of a kinetically sound velocity. As an extreme limiting example, we highlight

Case D: $\gamma_{1}=0, \gamma_{5}= \pm \frac{1}{2} \sqrt{\frac{b}{1-b}}$. In this case

$$
u_{D}^{n+\frac{1}{2}}= \pm \frac{1}{\sqrt{2 \alpha m d t}} \beta^{n+1} .
$$

This definition produces the correct kinetic energy, but is clearly not an appropriate definition of a meaningful velocity since it is void of any information about the associated trajectory $r^{n}$. Instead, at each time step, a random value is chosen from the Maxwell-Boltzmann Gaussian distribution, and simply assigned to the velocity variable.

Given that the velocity definitions of this paper are all built on the GJF trajectory, we retain the configurational Einstein diffusion result of Eq. (12) for any of the above choices of velocities. The corresponding GreenKubo calculations for $f=0$ using the derived two-point velocities can be found in Appendix B. The results show that the discrete-time Riemann sums allow for correct, and time step independent diffusion results if the rightRiemann sum is chosen for Case A (as also derived in Ref. [19]), and if the trapezoidal sum is chosen for Case B. A Green-Kubo expression for Case $\mathrm{C}$ also exists, but it is not given by one of the three traditional discrete-time
Riemann sums.

We also note that the evaluation of a correct GreenKubo value for diffusion in a flat potential $f \equiv 0$ is neither a guarantee for correct Green-Kubo results in systems where $f \neq 0$, nor is it necessarily a good indicator of the quality of kinetic measures for curved potentials. For example, the GJF on-site velocity, $v^{n}$ (5), produces the correct Green-Kubo result [see Eq. (A4)]; yet, it also produces a depressed kinetic energy (15). In general, it is the Einstein definition Eq. (12) that determines the actual diffusion, since this expression is a configurational measure for the actual square distance an object has been displaced over a given time. The simple Green-Kubo results shown in the Appendices are merely indicators of consistency between kinetic and configurational properties of a freely diffusing particle.

\section{NUMERICAL SIMULATIONS}

In order to validate the kinetic features obtained for the velocities presented in this paper, we conduct the same kind of molecular simulations that was used to validate Case A along with showing detailed comparisons with the well-known BBK [8] and PBS [9] methods in Ref. [19]. The system consists of $N=864$ atoms, each with mass $m$, in a cubic simulation cell with volume $V$ and periodic boundary conditions at a normalized pressure of approximately 0.1 . The interaction potential $E_{p}(r)$ is a splined, short range Lennard-Jones potential given by

$$
\frac{E_{p}(|r|)}{E_{0}}= \begin{cases}\left(\frac{|r|}{r_{0}}\right)^{-12}-2\left(\frac{|r|}{r_{0}}\right)^{-6} & , 0<|r| \leq r_{s} \\ \frac{a_{4}}{E_{0}}\left(|r|-r_{c}\right)^{4}+\frac{a_{8}}{E_{0}}\left(|r|-r_{c}\right)^{8} & , r_{s}<|r|<r_{c} \\ 0 & , r_{c} \leq|r|\end{cases}
$$

$r$ being a coordinate between a pair of particles. The spline parameters are given by

$$
\begin{aligned}
\frac{r_{s}}{r_{0}} & =\left(\frac{13}{7}\right)^{1 / 6} \approx 1.108683 \\
\frac{r_{c}}{r_{0}} & =\frac{r_{s}}{r_{0}}-\frac{32 E_{p}\left(r_{s}\right)}{11 E_{p}^{\prime}\left(r_{s}\right) r_{0}} \approx 1.959794 \\
a_{4} & =\frac{8 E_{p}\left(r_{s}\right)+\left(r_{c}-r_{s}\right) E_{p}^{\prime}\left(r_{s}\right)}{4\left(r_{c}-r_{s}\right)^{4}} \\
a_{8} & =-\frac{4 E_{p}\left(r_{s}\right)+\left(r_{c}-r_{s}\right) E_{p}^{\prime}\left(r_{s}\right)}{4\left(r_{c}-r_{s}\right)^{8}} .
\end{aligned}
$$

The potential $E_{p}(r)$ has minimum $-E_{0}$ at $|r|=r_{0}$, and is smoothly splined between the inflection point of the Lennard-Jones potential and zero with continuity through the second derivative at $|r|=r_{s}$, and continuity through the third derivative at $|r|=r_{c}$.

With these characteristic parameters, time is normalized to the inverse of the characteristic frequency $\omega_{0}=\sqrt{E_{0} / m r_{0}^{2}}$. Two characteristically different temperatures are tested; $k_{B} T=0.3 E_{0}$, which results in a stable fcc (face centered cubic) crystal at a volume of $V=617.2558 r_{0}^{3}$, and $k_{B} T=0.7 E_{0}$, which results in a liquid state at volume $V=824.9801 r_{0}^{3}$. We show results for three different friction coefficients: $\alpha=1 m \omega_{0}$, 


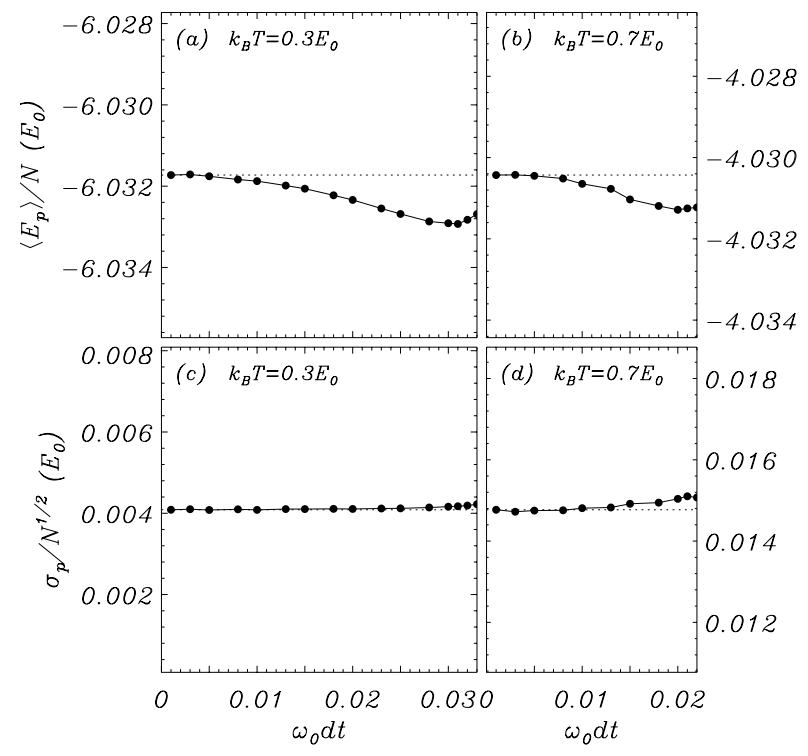

FIG. 1: Statistical averages of potential energy $\left\langle E_{p}\right\rangle$ (from Eq. (52) ) (a) and (b), and its standard deviation $\sigma_{p}$ (from Eq. (53) (c) and (d) as a function of reduced time step $\omega_{0} d t$ for $\alpha=1 m \omega_{0}$. (a) and (c) show results for a crystalline fcc state at $k_{B} T=0.3 E_{0}$; (b) and (d) show results for a liquid state at $k_{B} T=0.7 E_{0}$. Horizontal dotted lines indicate the results for small $\omega_{0} d t=0.001$.

$\alpha=10 m \omega_{0}$, and $\alpha=100 m \omega_{0}$. Notice that while these values seem high at first glance, they should be compared to the friction $\alpha_{0}=m \sqrt{E_{p}^{\prime \prime}\left(r_{0}\right) / m} \approx \sqrt{60} m \omega_{0}$ relevant to the simulated density and characteristic collision distances. From this comparison we conclude that the simulated friction coefficients represent damping values ranging from underdamped oscillatory to slightly overdamped behavior.

For each simulated temperature, friction value, and time step, we calculate statistical averages over one trajectory of reduced time of $\omega_{0} \Delta t=2 \times 10^{5}$, after the system has equilibrated for at least the same time before statistics is acquired. Data for all four velocities, the onsite GJF velocity (17), and Cases A (42), B (43), and $\mathrm{C}$ (44), are accumulated such that the displayed kinetic results for the different velocities on Figures 2 , 4 and 6 can be directly compared. The corresponding acquisition of the configurational statistics is shown on Figures 1, 3 , and 5 .

As expected from previous investigations 15, 19, 24, 25, 28, 29] of the GJF method, the configurational statistics is excellent (see Figures 1, 3, and 5). The displayed quantities are derived from the potential energy $E_{p}^{n}$ of the $N$-particle system

$$
E_{p}^{n}=\sum_{i=1}^{N} \sum_{j>i}^{N} E_{p}\left(\left|r_{i}^{n}-r_{j}^{n}\right|\right),
$$

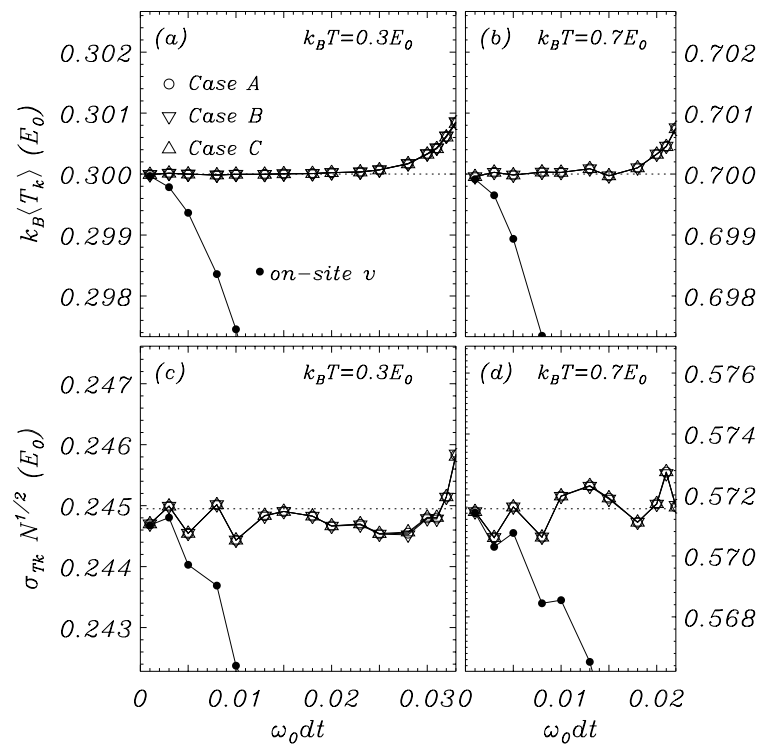

FIG. 2: Statistical averages of the kinetic temperature (from Eq. (55) ) (a) and (b), and their standard deviations (from Eq. (57) ) (c) and (d) as a function of reduced time step $\omega_{0} d t$ for $\alpha=1 m \omega_{0}$. (a) and (c) show results for a crystalline fcc state at $k_{B} T=0.3 E_{0} ;(\mathrm{b})$ and (d) show results for a liquid state at $k_{B} T=0.7 E_{0}$. Horizontal dotted lines indicate the exact, continuous time results,$\left\langle T_{k}\right\rangle^{*}$ and $\sigma_{T_{k}}^{*}$, given by Eqs. (56) and (58). Results shown for four velocity definitions: GJF on-site $v$, and velocities of Cases A, B, C.

from which we calculate the average

$$
\left\langle E_{p}\right\rangle=\left\langle E_{p}^{n}\right\rangle
$$

and the temporal fluctuations $\sigma_{p}$

$$
\sigma_{p}=\sqrt{\left\langle\left(E_{p}^{n}\right)^{2}\right\rangle-\left\langle E_{p}^{n}\right\rangle^{2}} .
$$

It is apparent that the calculated quantities are, with very good approximation, time step independent in the entire range of stability, with a slight decreasing trend for increasing time steps in the average of the potential energy, and a slight increase in its fluctuations. These deviations are most prominent for low friction values, but are minor on a relative scale. Since linear analysis of the method shows that no configurational sampling should deviate from expectations at any stable time step (see Eq. (14)), it follows that the observed deviations are a result of the nonlinearities in the interaction potential, and not a result of inherent systematic algorithmic inaccuracies [27]. Notice that the configurational behavior is independent of the specific velocity one may associate with the GJF method. Thus, the data shown in Figs. 1, 3. and 5 represent the use of any velocity, including Cases $\mathrm{A}, \mathrm{B}$, and $\mathrm{C}$.

The kinetic measures, which are the point of interest to this presentation (see Figures 2, 4. and 6), display 




FIG. 3: Statistical averages of potential energy $\left\langle E_{p}\right\rangle$ (from Eq. (52) ) (a) and (b), and its standard deviation $\sigma_{p}$ (from Eq. (53) (c) and (d) as a function of reduced time step $\omega_{0} d t$ for $\alpha=10 m \omega_{0}$. (a) and (c) show results for a crystalline fcc state at $k_{B} T=0.3 E_{0}$; (b) and (d) show results for a liquid state at $k_{B} T=0.7 E_{0}$. Horizontal dotted lines indicate the results for small $\omega_{0} d t=0.001$.

excellent time step independence, as one would expect from the analysis above since these investigated velocities are engineered to produce time step independence in their calculated kinetic energy. Specifically, we investigate the kinetic energy $E_{k}^{n+\frac{1}{2}}$ and temperature $T_{k}^{n+\frac{1}{2}}$ of the $N$ particle system at time $t_{n+\frac{1}{2}}$ :

$$
E_{k}^{n+\frac{1}{2}}=\frac{1}{2} \sum_{i=1}^{N} m\left(u^{n+\frac{1}{2}}\right)^{2}=\frac{3 N}{2} k_{B} T_{k}^{n+\frac{1}{2}},
$$

for which the temporal averages are expected to have the relationship

$$
\left\langle E_{k}\right\rangle=\left\langle E_{k}^{n+\frac{1}{2}}\right\rangle=\frac{3 N}{2} k_{B}\left\langle T_{k}^{n+\frac{1}{2}}\right\rangle=\frac{3 N}{2} k_{B}\left\langle T_{k}\right\rangle
$$

are expected to have the continuous-time

$$
\left\langle E_{k}\right\rangle^{*}=\frac{3 N}{2} k_{B}\left\langle T_{k}\right\rangle^{*}=\frac{3 N}{2} k_{B} T .
$$

We also calculate the temporal fluctuations $\sigma_{E_{k}}$ iand $\sigma_{T_{k}}$ in $E_{k}^{n+\frac{1}{2}}$ and $T_{k}^{n+\frac{1}{2}}$, respectively:

$$
\sigma_{E_{k}}=\sqrt{\left\langle\left(E_{k}^{n+\frac{1}{2}}\right)^{2}\right\rangle-\left\langle E_{k}^{n+\frac{1}{2}}\right\rangle^{2}}=\frac{3 N}{2} k_{B} \sigma_{T_{k}},
$$

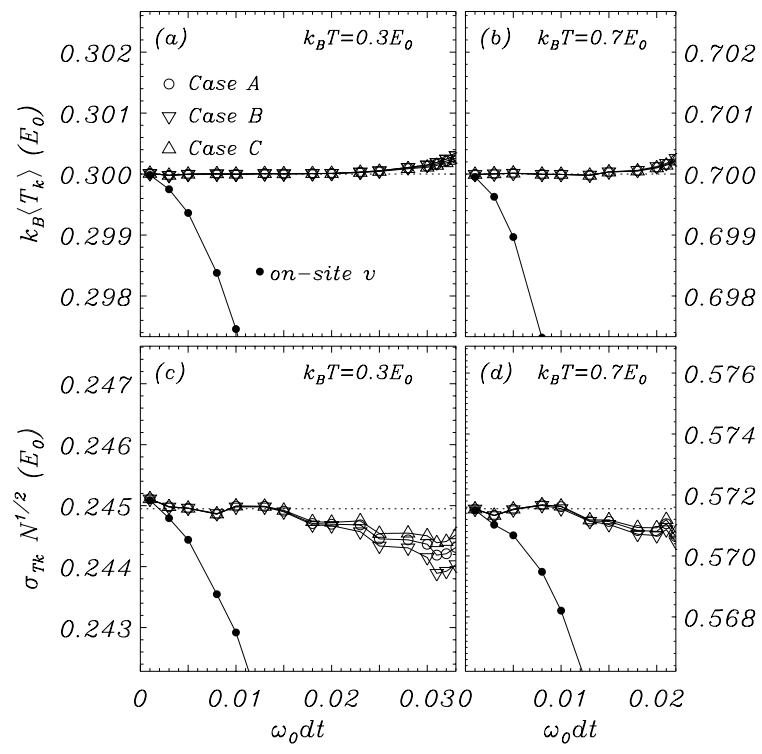

FIG. 4: Statistical averages of the kinetic temperature (from Eq. (55) ) (a) and (b), and their standard deviations (from Eq. (57) (c) and (d) as a function of reduced time step $\omega_{0} d t$ for $\alpha=10 m \omega_{0}$. (a) and (c) show results for a crystalline fcc state at $k_{B} T=0.3 E_{0} ;(\mathrm{b})$ and (d) show results for a liquid state at $k_{B} T=0.7 E_{0}$. Horizontal dotted lines indicate the exact, continuous time results,$\left\langle T_{k}\right\rangle^{*}$ and $\sigma_{T_{k}}^{*}$, given by Eqs. (56) and (58). Results shown for four velocity definitions: GJF on-site $v$, and velocities of Cases A, B, C.

where, in continous time, these fluctuations are expected to yield

$$
\sigma_{E_{k}}^{*}=\frac{3 N}{2} k_{B} \sigma_{T_{k}}^{*}=\sqrt{\frac{3}{2}} k_{B} T \sqrt{N} .
$$

The simulated quantities for the three highlighted cases of velocities, Cases A, B, and C, are shown in Figs. 2, 4, and 6 as markers (Eqs. (54) and (57)) and dotted horizontal lines (Eqs. (55) and (58)) for reference. We also show the comparable results when using the on-site GJF velocity $v^{n}$. The overall impression of the simulation results for the kinetic measures is that the two-point velocities behave largely in agreement with the analytical expectations in the entire stability range of the simulations. This is true for both simulated temperatures and states of matter. It is noticeable that Cases A, B, and C behave nearly identically, except for the high friction value, where Case B (the maximally scaled velocity) deviates from the two other definitions in the fluctuation measure. However, we notice that this deviation is minor. It is here important to emphasize that the kinetic results for the different velocity definitions shown in Figs. 2, 4, and 6 are derived from the same simulation. This explains why the fluctuations in, e.g., Fig. 2 $\mathrm{d}$, are identical for Cases A, $\mathrm{B}$, and $\mathrm{C}$, since for low friction the three definitions are nearly identical. This also means that the deviations between the behavior of the three two-point velocities seen 


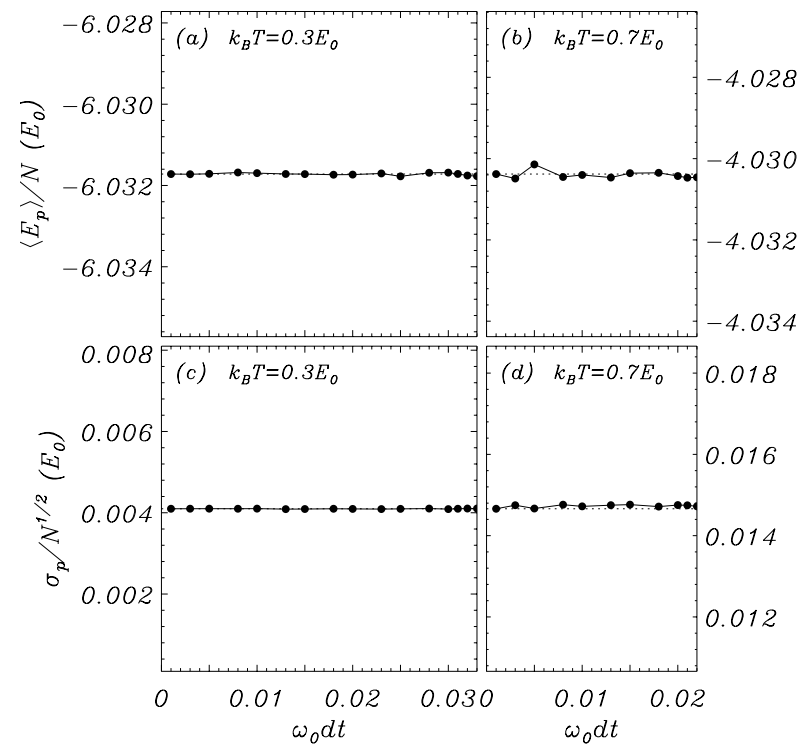

FIG. 5: Statistical averages of potential energy $\left\langle E_{p}\right\rangle$ (from Eq. (52) ) (a) and (b), and its standard deviation $\sigma_{p}$ (from Eq. (53) (c) and (d) as a function of reduced time step $\omega_{0} d t$ for $\alpha=100 m \omega_{0}$. (a) and (c) show results for a crystalline fcc state at $k_{B} T=0.3 E_{0}$; (b) and (d) show results for a liquid state at $k_{B} T=0.7 E_{0}$. Horizontal dotted lines indicate the results for small $\omega_{0} d t=0.001$.

in, e.g., Fig. 6re are a true reflection of differences between the velocities. We have further validated that reasonable choices of $\gamma_{1}<1$ also produce reliable results. Specifically, the cases $b \gamma_{1}=\sqrt{|a|}$ (the case for which velocity attenuation is equally partitioned over the two half time steps - see Eq. (39)-(41)), and $b \gamma_{1}=|a|$ (the case for which velocity attenuation is exclusively assigned to the first half of the time step) both yield results nearly indistinguishable from Cases A and C. We have omitted the display of these results in the figures for visual simplicity. The on-site GJF velocity is shown for comparison, and it is clear that in contrast to the velocities highlighted in this paper, the deviation for the on-site velocity is much more pronounced and, moreover, the error increases with the integration time-step.

\section{DISCUSSION}

Inspired by the discovery of a velocity definition that can produce accurate kinetic statistics in conjunction with the GJF thermostat, we have here analyzed all possible finite difference velocity definitions that may accompany the GJF trajectory. We draw two important conclusions: First, that it is not possible to identify a meaningful on-site velocity such that the kinetic measures of thermodynamics can be time step independent. Second, that there exist an infinite number of two-point velocities such that the kinetic energy is correctly evaluated. Hav-

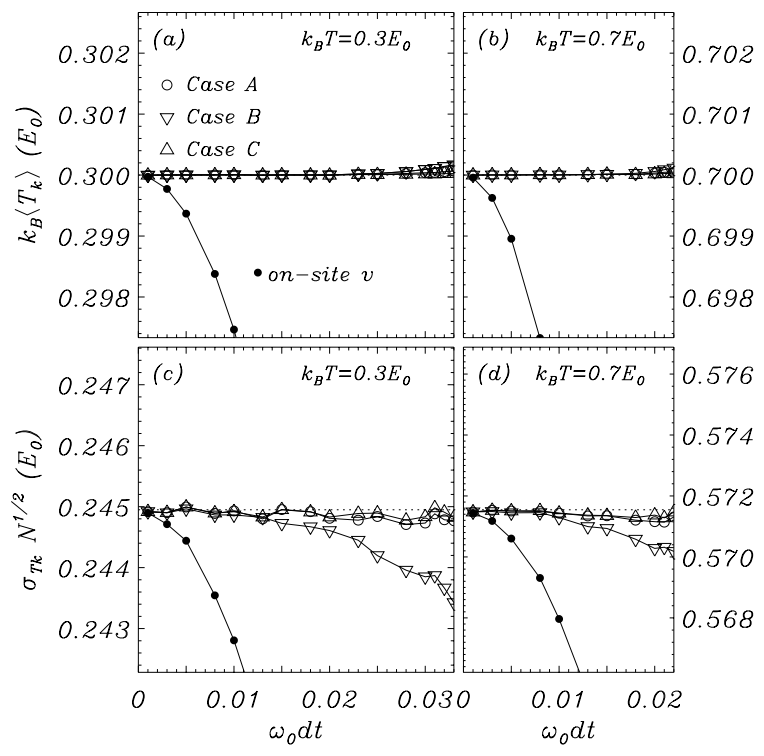

FIG. 6: Statistical averages of the kinetic temperature (from Eq. (55) ) (a) and (b), and their standard deviations (from Eq. (57) ) (c) and (d) as a function of reduced time step $\omega_{0} d t$ for $\alpha=100 m \omega_{0}$. (a) and (c) show results for a crystalline fcc state at $k_{B} T=0.3 E_{0} ;(\mathrm{b})$ and (d) show results for a liquid state at $k_{B} T=0.7 E_{0}$. Horizontal dotted lines indicate the exact, continuous time results,$\left\langle T_{k}\right\rangle^{*}$ and $\sigma_{T_{k}}^{*}$, given by Eqs. (56) and (58). Results shown for four velocity definitions: GJF- on-site $v$, and velocities of Cases A, B, C.

ing identified this family of velocities, we have included them in the GJF formalism and introduced the general LF GJF algorithm, which is the leap-frog form Eqs. (35)(38). We have additionally written the set of methods in a convenient and compact form of Eqs. (39)-(41), that includes any of the defined velocities together with the native GJF on-site velocity such that the method is entirely contained with operations pertaining only to a single time step. The set of kinetically sound velocities is parameterized by a single parameter $\left(\gamma_{1}\right)$, and we have highlighted three choices that seem either mathematically or physically attractive within the physical limitations to values of $\gamma_{1}$.

Molecular simulations of Lennard-Jones test cases have confirmed the predicted features of the new set of velocities, which seem to display very good time step independent behavior throughout the stability ranges for the time step. All three highlighted velocity definitions (which are for $\gamma_{1} \geq 1$ ) show near identical statistical behavior, except for the most amplitude-distorted velocity, which exhibits some minor deviations in its fluctuations for large time steps. Additionally, we have verified that also two other reasonable choices for $\gamma_{1}<1$ exhibit results similar to the cases highlighted in the figures.

It is our hope that the complete set of defined GJF velocities will be further explored such that a more complete understanding of the different definitions can be de- 
veloped and refined for a variety of applications. We have specifically validated a select few of the possible velocity definitions, but there may very well be other choices that are more appealing in some instances. The cases of immediate interest are Cases A and C. Case A is the true half-step velocity that is correctly cross correlated with the spatial degree of freedom [29], but does not give correct drift velocity. Case $\mathrm{C}$ gives correct drift velocity, but is not a half-step velocity, since it is not symmetrically evaluated relative to the spatial coordinate $r^{n}$. These features suggest that Case A should be preferred for equilibrium simulations with diffusive transport, while Case $\mathrm{C}$ could be preferred for non-equilibrium simulations involving drift or ballistic transport.

Our simulations demonstrated robustness of all highlighted definitions in standard Molecular Dynamics systems. The many possibilities must be explored by the community that conduct Langevin dynamics simulations so that the breadth of applications, limitations, and conditions can be adequately tested.
[1] M. P . Allen, D. J. Tildesley, Computer Simulation of Liquids, Oxford University Press, Inc., New York, 1989.

[2] D. Frenkel and B. Smit, Understanding Molecular Simulations: From Algorithms to Applications, (Academic Press, San Diego, 2002).

[3] D. C. Rapaport, The Art of Molecular Dynamics Simulations, (Cambridge University Press, Cambridge, 2004).

[4] L. Verlet, Phys. Rev. 159, 98 (1967).

[5] S. Nosé, J. Chem. Phys. 81, 511 (1984).

[6] W. G. Hoover, Phys. Rev. A 31, 1695 (1985).

[7] T. Schneider and E. Stoll, Phys. Rev. B 17, 1302 (1978).

[8] A. Brünger, C. L. Brooks, and M. Karplus, Chem. Phys. Lett. 105, 495 (1984).

[9] R. W. Pastor, B. R. Brooks, and A. Szabo, Mol. Phys. 65, 1409 (1988).

[10] W. F. van Gunsteren, H. J. C. Berendsen, Mol. Simul. 1, 173, (1988).

[11] R. J. Loncharich, B. R. Brooks, and R. W. Pastor, Biopolymers 32, 523 (1992).

[12] E. Vanden-Eijnden, G. Ciccotti, Chem. Phys. Lett. 429, 310 (2006).

[13] S. Melchionna, J. Chem. Phys. 127, 044108 (2007).

[14] B. Leimkuhler, C. Matthews, Appl. Math. Res. Express 2013, 34 (2012).

[15] N. Grønbech-Jensen and O. Farago, Mol. Phys. 111, 983 (2013).

[16] E. Paquet, H. L. Viktor, BioMed Res. Int., 183918 (2015).

[17] P. Langevin, C. R. Acad. Sci. Paris 146, 530 (1908).

[18] G. Parisi, Statistical Field Theory, (Addison-Wesley, Menlo Park, 1988).

[19] L. F. G. Jensen and N. Grønbech-Jensen, Mol. Phys. 117, 2511 (2019).

[20] O. G. Jepps, G. Ayton, and D. J. Evans, Phys. Rev. E 62, 4757 (2000).

[21] G. Rickayzena and J. G. Powles, J. Chem. Phys. 114, 4333 (2001).

[22] P. J. Davis, B.A. Dalton, and T. Morishita, Phys. Rev. E 86, 056707 (2012).

[23] N. Jackson, J. Miguel Rubi, and Fernando Bresme, Mol. Sim. 42, 1214 (2016).

[24] N. Grønbech-Jensen, N. R. Hayre, and O. Farago, Comput. Phys. Commun. 185, 524 (2014).

[25] E. Arad, O. Farago, and N. Grønbech-Jensen, Isr. J. Chem. 56, 629 (2016).

[26] O. Farago, Physica A 534, 122210 (2019).

[27] L. F. G. Jensen and N. Grønbech-Jensen, Comput. Phys. Commun. (2019), DOI: 10.1016/j.cpc.2019.107011. arXiv:1902.02338.

[28] J. Finkelstein, G. Fiorin, and B. Seibold, Mol. Phys.
(2019), DOI: 10.1080/00268976.2019.1649493.

[29] N. Grønbech-Jensen, Mol. Phys. (2019), DOI: 10.1080/00268976.2019.1662506. arXiv:1909.04380.

[30] M. S. Green, Chem. Phys. 22, 398 (1954); R. Kubo, J. Phys. Soc. Jpn. 12, 570 (1957).

\section{Appendix A: Green-Kubo diffusion using the GJF on-site velocity $v^{n}$ for $f=0$}

The Green-Kubo equivalent of the Einstein expression for diffusion Eq. (12) is in discrete-time calculated from the autocorrelation $\left\langle v^{q+n} v^{q}\right\rangle_{q}$ of the GJF velocity Eq. (5), where

$$
v^{q}=a^{q} v^{0}+\frac{b}{m} \sum_{k=1}^{q} a^{q-k} \beta^{k} .
$$

Assuming that $v^{q}$ is a well-equilibrated velocity $\left(|a|^{q} \rightarrow\right.$ $0)$, the velocity autocorrelation in discrete-time reads

$$
\begin{aligned}
\left\langle v^{q+n} v^{q}\right\rangle= & \frac{b^{2}}{m^{2}}\left\langle\sum_{k=1}^{q+n} a^{q+n-k} \beta^{k} \sum_{l=1}^{q} a^{q-l} \beta^{l}\right\rangle \\
& \stackrel{q \rightarrow \infty}{\longrightarrow} a^{n} \frac{k_{B} T}{m} .
\end{aligned}
$$

The Green-Kubo diffusion coefficient, $D_{G K}$, is evaluated by the continuous-time expression [30]

$$
D_{G K}=\int_{0}^{\infty}\langle v(t+s) v(t)\rangle_{t} d s .
$$

However, as pointed out in Ref. [19], this definition is somewhat ambiguous in discrete time since different Riemann sums can be legitimately considered as approximations to the integral in (A3) for $d t>0$. The result for the trapezoidal sum is:

$$
\begin{aligned}
D_{G K} & =\sum_{k=0}^{\infty}\left\langle v^{q+k} v^{q}\right\rangle d t=\frac{k_{B} T}{m} d t\left(\frac{1}{2}+\sum_{k=1}^{\infty} a^{k}\right) \\
& =\frac{k_{B} T}{\alpha} .
\end{aligned}
$$

Thus, if the Green-Kubo integral is approximated by the trapezoidal Riemann sum, then we obtain the correct diffusion coefficient $D_{G K}$ consistent with the correct 
configurational value, $D_{E}$, given by Eq. (12). However, we could have used other Riemann approximations that converge to the correct results $D_{E}$ for $d t \rightarrow 0$, but do not exactly match it for $d t>0$. For example, the leftRiemann sum gives $D_{G K}=D_{E} / b>D_{E}$, while the rightRiemann sum gives $D_{G K}=D_{E}(a / b)<D_{E}$. Notice how sensitive the choice of the discrete approximation to the integral is by comparing the right-Riemann sum, which can yield even a nonsensical negative diffusion constant for $d t>d t_{a}$ (i.e., $a<0$ ), to the trapezoidal approximation, which in this case is both time step independent and correct.

\section{Appendix B: Green-Kubo diffusion using a two-point velocity $u^{n+\frac{1}{2}}$ for $f=0$}

For evaluating the Green-Kubo expression (A3) using the derived two-point velocity, we will need the discretetime velocity autocorrelation function. For that purpose, we define an initial condition at $t_{\frac{1}{2}}$, which we insert into Eq. (35) for $f=0$. Iterating the equation $q$ times yields

$$
\begin{aligned}
u^{q+\frac{1}{2}}= & a^{q} u^{\frac{1}{2}}+a^{q-1} \frac{\Gamma_{4}}{2 m} \beta^{1}+\frac{\Gamma_{5}}{2 m} \beta^{q+1} \\
& +\sum_{k=0}^{q-2}\left(\frac{\Gamma_{4}}{2 m}+a \frac{\Gamma_{5}}{2 m}\right) a^{k} \beta^{q-k} .
\end{aligned}
$$

After lengthy, but trivial, calculations, we arrive at the compact form for the velocity autocorrelation in the limit $q \rightarrow \infty$ (i.e., after the memory of the initial velocity is lost)

$\left\langle u^{q+\frac{1}{2}+n} u^{q+\frac{1}{2}}\right\rangle=\frac{k_{B} T}{m}\left[a^{n}+a^{n-1} \Gamma_{4} \Gamma_{5} \frac{\alpha d t}{2 m}\left(1-\delta_{n, 0}\right)\right]$.

This expression can be used to approximate the GreenKubo expression for diffusion by a Riemann sum. As was discussed in Ref. [19] and Appendix A we can justify any Riemann sum of the Green-Kubo integral in Eq. A3 with a discretization of $d t$. The three obvious choices, left-Riemann $D_{l R}$, trapezoidal $D_{t r}$, and right-Riemann $D_{r R}$ sums yield

$$
\begin{aligned}
D_{l R} & =\frac{k_{B} T}{m} d t\left[1+\frac{1}{1-a}\left(a+\Gamma_{4} \Gamma_{5} \frac{\alpha d t}{2 m}\right)\right] \\
D_{t r} & =\frac{k_{B} T}{m} d t\left[\frac{1}{2}+\frac{1}{1-a}\left(a+\Gamma_{4} \Gamma_{5} \frac{\alpha d t}{2 m}\right)\right] \\
D_{r R} & =\frac{k_{B} T}{m} d t \frac{1}{1-a}\left(a+\Gamma_{4} \Gamma_{5} \frac{\alpha d t}{2 m}\right)
\end{aligned}
$$

where $D_{r R} \leq D_{t r} \leq D_{l R}$. When selecting $\gamma_{1}$ and $\gamma_{5}$, an additional consideration in determining if a velocity is meaningful may be that the corresponding Green-Kubo diffusion expressions can yield a result such that $D_{E} \in$ $\left[D_{r R}, D_{l R}\right]$. Not all choices of $\gamma_{1}$ yield a velocity variable satisfying this criterion. Therefore, we define a parameter $0 \leq \mathcal{C} \leq 1$, which can determine if a given velocity (as defined by $\gamma_{1}$ and $\gamma_{5}$ ) can produce $D_{E} \in\left[D_{r R}, D_{l R}\right]$, i.e., if there exists a $\mathcal{C} \in[0,1]$ for the given method such that

$$
D_{E}=\frac{k_{B} T}{\alpha}=D_{r R}+\mathcal{C} \frac{k_{B} T}{m} d t .
$$

From Eq. (B3) the value of $\mathcal{C}$ for which Eq. (B6) is true is given by

$$
\mathcal{C}=\frac{1}{2}-\frac{\Gamma_{4} \Gamma_{5}}{2 b}
$$

and the condition for a given velocity to have a GreenKubo diffusion value within the acceptable range is then

$$
-b \leq \Gamma_{4} \Gamma_{5} \leq b .
$$

It is straightforward to verify that the above highlighted cases A, B, and C satisfy this criterion for any value $0 \leq b \leq 1$. Specifically, the optimally scaled velocity, $u_{A}^{n+1 / 2}$ (42), gives the correct Einstein diffusion for $D_{r R}$ (as described in Ref. [19]), and the maximally scaled velocity, $u_{B}^{n+1 / 2}$ (43), gives the correct diffusion for $D_{t r}$. 